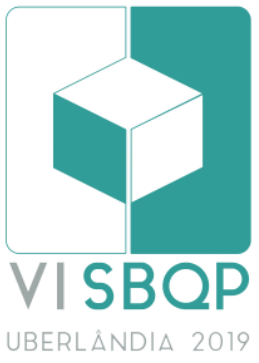

\title{
ESTUDO HIGROTÉRMICO NA AUTOCONSTRUÇÃO: SIMULAÇÕES E MEDIÇÕES EM CAMPO
}

\author{
DANTAS, André Luís \\ Universidade de Brasília, e-mail: andreldants@gmail.com \\ NUNES, Layane Soares \\ Universidade de Brasília, e-mail: layanecute@gmail.com \\ RIOS, Rafael Barbosa \\ Universidade de Brasília, e-mail: rafaelb.rios@gmail.com \\ ZANONI, Vanda Alice Garcia \\ Universidade de Brasília, e-mail: vandazanoni@unb.br
}

\begin{abstract}
RESUMO
A exposição das edificações aos agentes climáticos pode comprometer a durabilidade e vida útil dos sistemas que a compõem. As autoconstruções representam parte da realidade brasileira, no entanto, são pouco estudadas, principalmente quanto ao seu comportamento higrotérmico. Esse artigo tem o objetivo de analisar o comportamento higrotérmico da parede de alvenaria da fachada Leste de uma autoconstrução, localizada em Santa Maria- DF, durante 0 mês de janeiro. Foram realizadas simulações higrotérmicas no software WUFI Pro e medições em campo de umidade e temperatura com um microcontrolador Arduino Uno e sensores DHT22, posicionados no ambiente interno e externo da edificação. Observou-se que as simulações e as medições realizadas para o ambiente externo, quando comparadas, obtiveram variações pequenas, enquanto no ambiente interno, as medições apresentaram variações muito maiores que a simulação. Isso indica que o sistema construtivo adotado na autoconstrução é muito mais suscetível a variações higrotérmicas do que as previstas em simulação. Os resultados desta pesquisa são relevantes porque contribuem com a compreensão de uma realidade pouco estudada, como as autoconstruções, e auxiliam com as decisões de planejamento no âmbito das ações de melhoria habitacional e assistência técnica.
\end{abstract}

Palavras-chave: Desempenho higrotérmico, Simulação computacional, Medições em campo, Autoconstrução.

\begin{abstract}
The exposure of buildings to the climate agents may jeopardize the durability and the lifespan of the constructive systems that make it up. The auto constructions represents the Brazilian reality, but are, in the meantime, little studied. This article has the goal to observe the hygrothermal behavior of the eastern masonry wall of an auto construction during January, located in Santa Maria-DF. Hygrothermal simulations were developed using the software WUFI Pro, and also field monitoring using a micro controller Arduino Uno connected to DHT22 sensors, positioned in the indoor and outdoor faces. It was observed that the simulation and the monitoring for the outdoor conditions obtained few variations, while in the indoors, the monitoring obtained variations bigger than the simulations. This indicates that the auto constructions are susceptible to hygrothermal variations bigger than the values recommended in national and international standards. This research may contribute to the understanding of a little studied subject, which is the auto
\end{abstract}

DANTAS, A. L.; NUNES, L. S.; RIOS, R. B.; ZANONI, V. A. G. Estudo higrotérmico na autoconstrução: simulações e medições em campo. In: SIMPÓSIO BRASILEIRO DE QUALIDADE DO PROJETO NO AMBIENTE CONSTRUÍDO, 6., 2019, Uberlândia. Anais... Uberlândia: PPGAU/FAUeD/UFU, 2019. p. 671-678. DOI https://doi.org/10.14393/sbqp19062. 
constructions and may assist planning decisions in the scope of actions leaded to habitation improvement and technical assistance.

Keywords: Hygrothermal performance, Computer simulation, Field monitoring, Auto construction.

\section{INTRODUÇÃO}

A prática da autoconstrução faz parte da realidade brasileira. Segundo uma pesquisa, realizada com 2.419 pessoas em todo o Brasil, 54\% da população economicamente ativa já construiu ou reformou imóvel residencial ou comercial. Desse grupo, $85,40 \%$ fizeram o serviço por conta própria ou com pedreiros e mestres de obras, amigos e parentes. Apenas $14,60 \%$ contratou arquitetos ou engenheiros (Pesquisa CAU/BR - Datafolha,12/10/2015).

Apesar dos impactos das atividades da autoconstrução em todo o país, que são executadas sem a presença de um profissional responsável, o comportamento dos sistemas construtivos adotados é pouco estudado, principalmente no que diz respeito ao seu desempenho higrotérmico.

Sabe-se que os agentes climáticos podem afetar a durabilidade e 0 desempenho dos sistemas construtivos de um edifício, conforme suas condições de exposição. Uma vez que as autoconstruções carecem da atuação de profissionais da construção, os requisitos e critérios normativos, como os da Norma de Desempenho NBR 15575, não são levados em conta no momento da construção, podendo acarretar prejuízos para o desempenho e vida útil dos sistemas construtivos da edificação.

A simulação computacional pode contribuir para compreensão das condicionantes climáticas que influenciam na vida útil dos sistemas construtivos, possibilitando a criação de modelos e avançando no entendimento do comportamento higrotérmico (ZANONI, 2015). Outro passo para compreender os danos que resultam da ação da umidade e da temperatura nas construções é o monitoramento em campo das condições internas e externas de umidade e temperatura (COELHO; SILVA; HENRIQUES, 2018). Quando o método de pesquisa permite associar os procedimentos de campo com as simulações, os dados coletados proporcionam maior sensibilidade e confiabilidade no entendimento dos fenômenos.

Neste contexto, esse estudo tem o objetivo de observar a ação da umidade e temperatura, internamente e externamente, em uma autoconstrução de alvenaria de tijolos cerâmicos furados localizada em Santa Maria - DF, por meio de simulações computacionais e medições em campo realizadas durante o mês de janeiro de 2019, visando a compreensão de uma realidade pouco estudada, como as autoconstruções, e as decisões de planejamento no âmbito das ações de melhoria habitacional e assistência técnica.

\section{MÉTODO}

O estudo foi desenvolvido por meio das seguintes etapas: revisão bibliográfica, coleta de dados por meio das medições em campo utilizando sensores de umidade e temperatura, simulação computacional higrotérmica e análises de dados coletados.

A simulação computacional foi feita no software de simulação higrotérmica WUFI Pro 6.2 (Wärme- Und Feuchtetransport Instationär - Transient Heat and 
Moisture Transport), que utiliza os arquivos climáticos em formato TMY (Typical Meteorological Year) com dados medidos pelo INMET (Instituto Nacional de Meteorologia). Os arquivos climáticos utilizados para a realização deste trabalho foram disponibilizados pelo LabEEE (Laboratório de Eficiência Energética em Edificações) nas versões 2016 e 2018 . A simulação visou calcular as condições de exposição aos agentes climáticos em uma determinada localidade durante um ano climático típico.

As medições em campo foram realizadas em uma habitação térrea autoconstruída, de alvenaria de tijolo cerâmico furado localizada na cidade de Santa Maria-DF, na Zona Bioclimática 4 (Figura 1). Durante as medições, a casa encontrava-se em uso regular, habitada por 4 pessoas. As renovações de ar aconteciam somente pelas janelas, não possuindo outros meios de acondicionamento de ar. Foram medidas quatro variáveis: umidade relativa do ar na superfície externa, umidade relativa do ar na superfície interna, temperatura na superfície externa e temperatura na superfície interna. As medições foram realizadas por meio de dois sensores DTH22 posicionados na face interna e externa da parede de alvenaria da fachada Leste (Figura 2). Os sensores foram conectados a um microcontrolador Arduino Uno que armazena e registra os dados a cada 10 minutos.
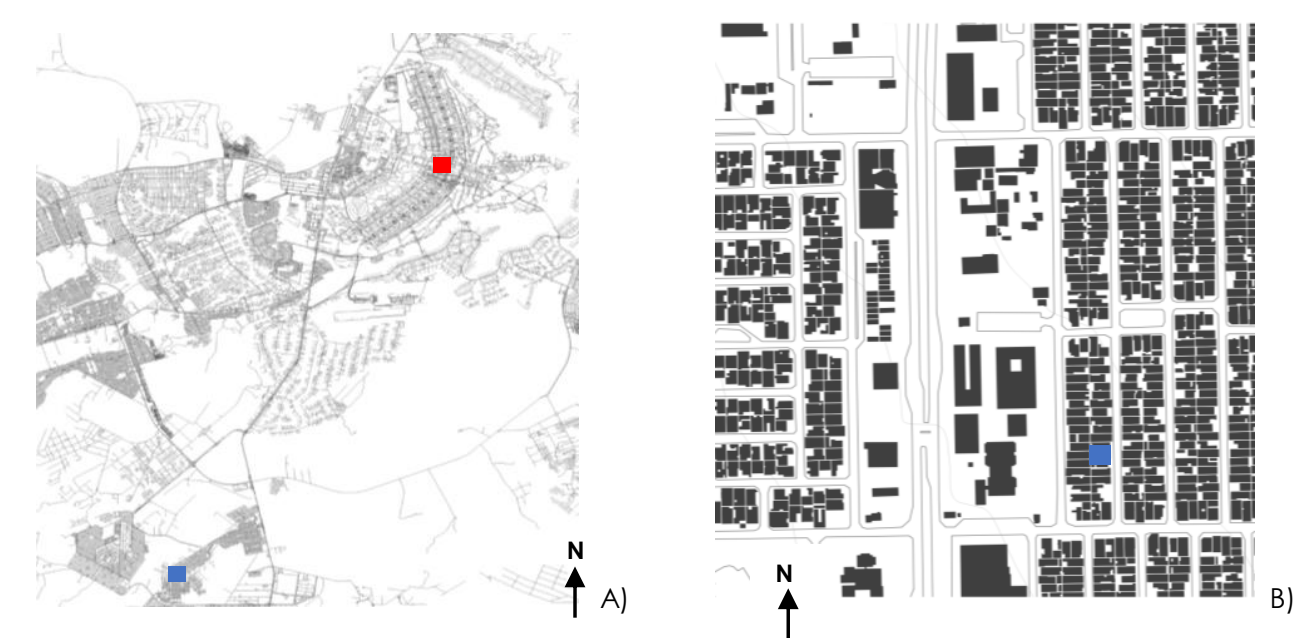

Figura 1 - Unidade habitacional autoconstruída: A) Localização em Brasília-DF; B) Localização na Região Administrativa de Santa Maria -

Fonte: Autores
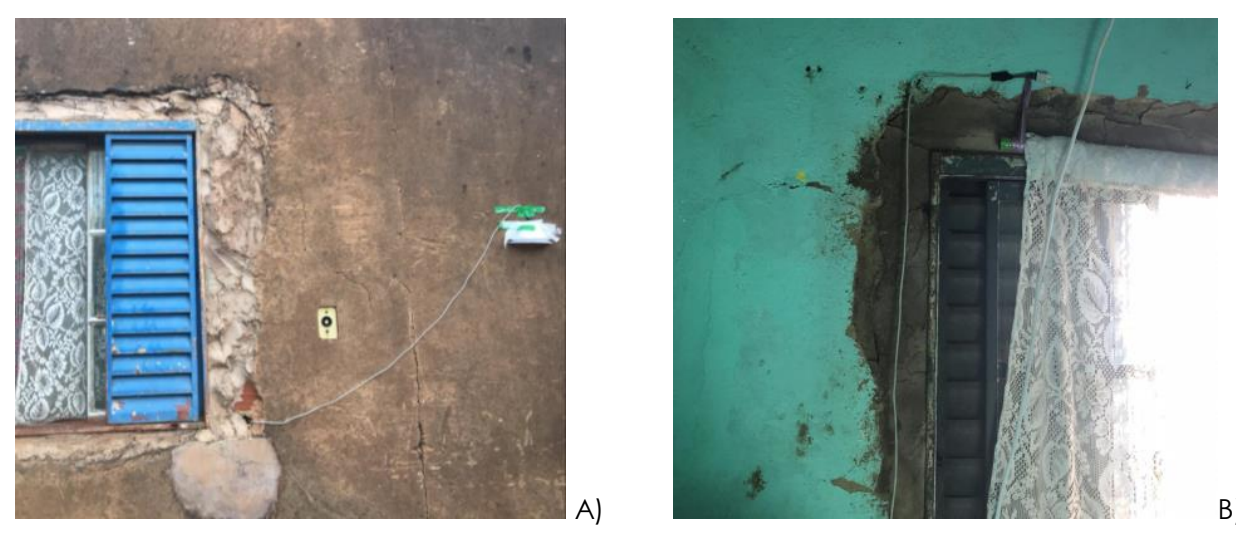

Figura 2 - Sensores DTH22: A) Posição do sensor na face externa da parede; B) Posição do sensor na face interna da parede - 
Uma vez coletados os dados pelos diferentes métodos, foi feito um cruzamento das informações obtidas na simulação com as medidas em campo. Esse cruzamento de dados permitiu uma análise de sensibilidade do que fora medido em campo com o arquivo climático, que apresenta valores horários para o mês de janeiro.

Para a simulação do sistema de vedação vertical da autoconstrução em questão, foi utilizada uma parede de alvenaria de tijolo cerâmico de $9 \mathrm{~cm}$ de espessura e argamassa de revestimento em ambas as faces, com $2 \mathrm{~cm}$ de espessura (Figura 3). As propriedades dos materiais adotados na simulação estão expostas no Quadro 1 e foram baseadas nas simulações desenvolvidas por Zanoni (2015) com os dados fornecidos pelo software WUFI (Quadro 1).

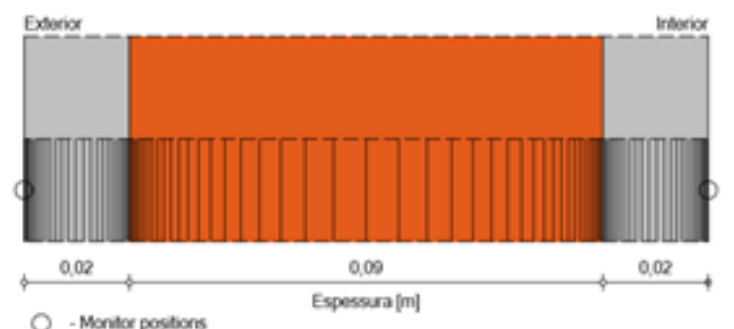

$\begin{array}{ll}\text { Argamassa de Revestimento } & 2 \mathrm{~cm} \\ \text { Tijolo Cerâmico } & 9 \mathrm{~cm} \\ \text { Argamassa de Revestimento } & 2 \mathrm{~cm}\end{array}$

Figura 3 - Caracterização do sistema construtivo simulado Fonte: WUFI, adaptado pelos autores

Quadro 1- Propriedades dos materiais componentes do elemento construtivo

\begin{tabular}{|c|c|c|c|c|c|c|c|c|c|c|c|c|c|}
\hline & & $\begin{array}{c}1 \\
\rho \\
\mathrm{kg} / \mathrm{m}^{3}\end{array}$ & $\begin{array}{c}2 \\
\varepsilon \\
\mathrm{m}^{3} / \mathrm{m}^{3}\end{array}$ & $\begin{array}{c}3 \\
\mathrm{C} \\
\mathrm{J} / \mathrm{kg} . \mathrm{K}\end{array}$ & & $\begin{array}{c}4 \\
\lambda \\
W / m . k\end{array}$ & & $\begin{array}{c}5 \\
\mu \\
{[-]}\end{array}$ & $\begin{array}{c}6 \\
\text { Wref } \\
\mathrm{kg} / \mathrm{m}^{3}\end{array}$ & $\begin{array}{c}7 \\
\text { Wsat } \\
\mathrm{kg} / \mathrm{m}^{3}\end{array}$ & $\begin{array}{c}8 \\
\text { Avalor } \\
\mathrm{kg} / \mathrm{m}^{2} \text {. } \\
\sqrt{\mathrm{s}}\end{array}$ & $\begin{array}{c}9 \\
\% / M .- \\
\%\end{array}$ & $\begin{array}{c}10 \\
\mathrm{~W} / \mathrm{m} \cdot \mathrm{K}\end{array}$ \\
\hline $\begin{array}{c}\text { Argamassa } \\
\text { de } \\
\text { Revestimente }\end{array}$ & & 1600 & 0,33 & 850 & & 0,7 & & 12 & 24 & 186 & 0,1667 & 3,7 & 0,0002 \\
\hline $\begin{array}{c}\text { Tijolo } \\
\text { Cerâmico }\end{array}$ & & 765 & 0,6 & 850 & & 0,158 & & 15 & 13 & 193 & 0,16 & 8 & 0,0002 \\
\hline Legenda: & 1 & \multicolumn{3}{|c|}{ Densidade de massa aparente } & م & $\mathrm{kg} / \mathrm{m}^{3}$ & 6 & \multicolumn{4}{|c|}{ Teor de umidade de referência } & Wret & $\mathrm{kg} / \mathrm{m}^{3}$ \\
\hline & 2 & \multicolumn{3}{|l|}{ Porosidade } & $\varepsilon$ & $\mathrm{m}^{3} / \mathrm{m}^{3}$ & 7 & \multicolumn{4}{|c|}{ Umidade de saturação livre } & Wsat & $\mathrm{kg} / \mathrm{m}^{3}$ \\
\hline & 3 & \multicolumn{4}{|c|}{ Calor especifico } & $\mathrm{J} / \mathrm{kak}$ & 8 & \multicolumn{4}{|c|}{ Coeficiente de absorção de água } & Axalor & $\mathrm{kg} / \mathrm{m}^{2}$. $\sqrt{\mathrm{s}}$ \\
\hline & 4 & \multicolumn{3}{|c|}{ Condutividade térmica } & $\lambda$ & $W / \cos k$ & 9 & \multicolumn{4}{|c|}{ Cond. térmica dependente da umidade } & $\% / M . \%$ & \% \\
\hline & 5 & \multicolumn{3}{|c|}{$\begin{array}{l}\text { Fator de resistência à difusão } \\
\text { de vapor de água }\end{array}$} & $\mu$ & {$[-]$} & 10 & \multicolumn{4}{|c|}{$\begin{array}{l}\text { Cond. térmica dependente da } \\
\text { temperatura }\end{array}$} & dceomel & W/onk \\
\hline
\end{tabular}

O tijolo adotado na simulação foi escolhido por conta da sua alta porosidade, acarretando um maior transporte de massa (umidade) pela parede, proporcionando um estudo mais realístico com as condições do sistema construtivo em análise. Justifica-se ao considerar que frequentemente os problemas encontrados em habitações autoconstruídas decorrem da utilização de materiais mais porosos e presença de umidade.

A altura do edifício simulado é outra variável importante, e no caso a edificação térrea estudada é classificada como edifício pequeno de até 10 metros de altura. Quanto maior a altura do edifício, maior será a velocidade 
do vento e a incidência de chuva dirigida. Em Brasília, o diagrama de chuva dirigida (Figura 4) indica o comportamento anual da chuva incidente, predominante nas fachadas Norte e Noroeste.

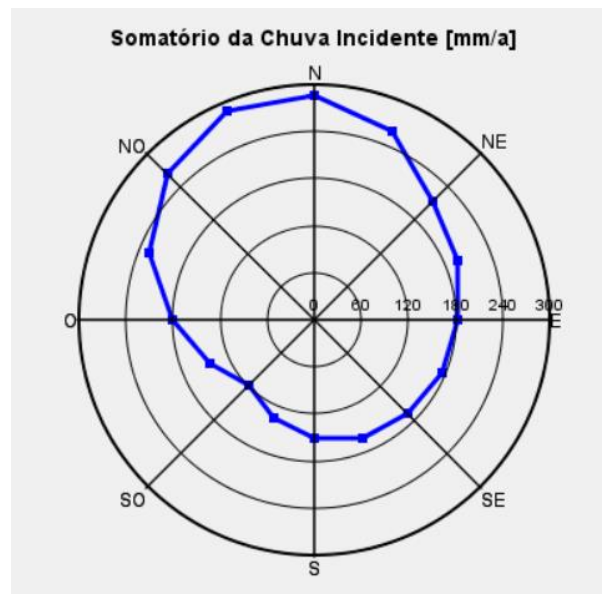

Figura 4 - Diagrama do somatório anual da chuva dirigida na cidade de Brasília Fonte: WUFI, adaptado pelos autores

A fachada Norte é aquela que concentra os maiores índices de chuva dirigida para o mês de janeiro. No entanto, nesta pesquisa de campo, foram coletados dados da fachada Leste, pois esta era a única exposta tanto à radiação solar quanto à chuva dirigida. De acordo com Zanoni (2015), a fachada Leste em janeiro em Brasília recebe cerca de 29,17 L/m² de chuva dirigida, pelo método descrito na norma ASHRAE 160.

\section{RESULTADO E DISCUSSÕES}

Os valores obtidos na simulação para o mês de janeiro estão expostos na Figura 5.

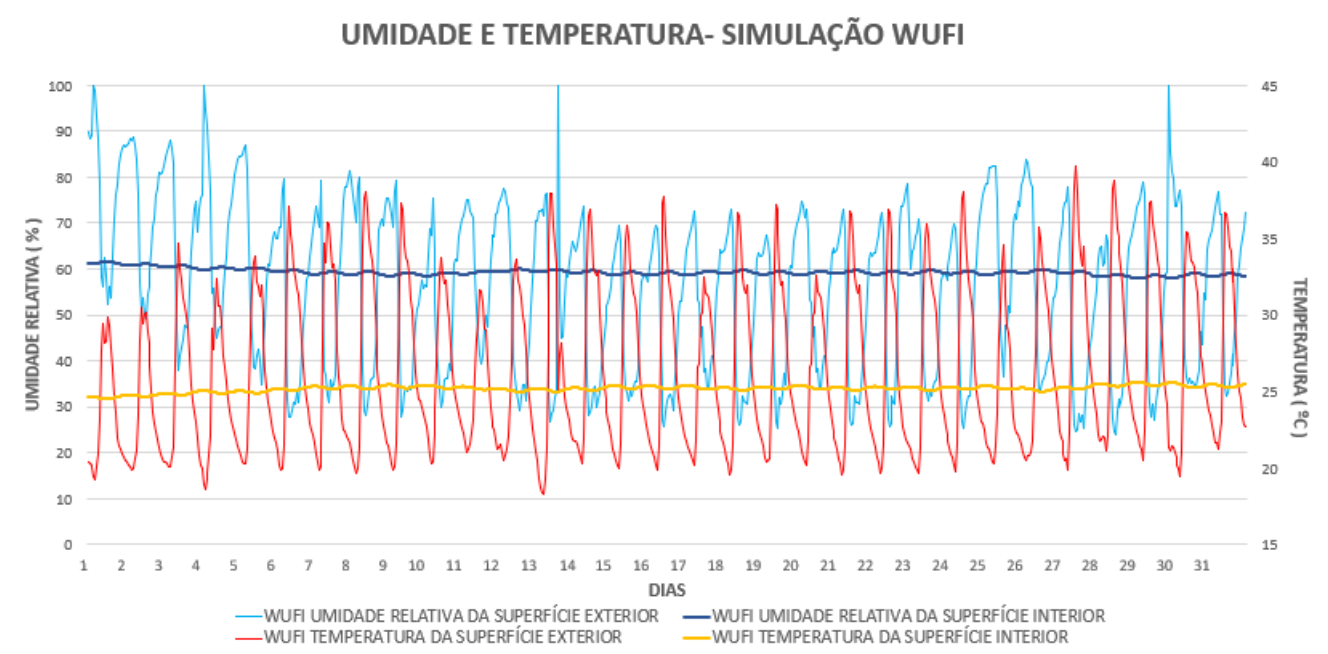

Figura 5 - Valores de simulação desenvolvida no software WUFI Pro Fonte: Autores

Os valores simulados de temperatura superficial exterior atingiram valores mínimos e máximos de $18,32^{\circ} \mathrm{C}$ e de $39,73^{\circ} \mathrm{C}$, respectivamente. Os valores de umidade relativa da superfície exterior variaram entre $23,9 \%$ a $99,9 \%$. Na 
superfície interna, os valores de temperatura variaram de $24,54{ }^{\circ} \mathrm{C}$ a $25,60{ }^{\circ} \mathrm{C}$, enquanto a umidade variou de $57,98 \%$ a $61,61 \%$.

Na simulação, os valores de umidade relativa e temperatura da superfície interior estabelecidos pelo WUFI variam muito pouco, ficando próximos de $25^{\circ} \mathrm{C}$ de temperatura e $60 \%$ de umidade relativa, uma vez que o modelo adotado pelo software estabelece uma condição estável de temperatura e umidade para o ambiente interno, em uma condição desejada de conforto.

Os valores obtidos nas medições em campo de temperatura estão expostos nas Figura 6, e os de umidade relativa estão expostos nas Figura 7.

TEMPERATURA - MEDIÇÕES DHT22 E DIA TÍPICO NBR 15575

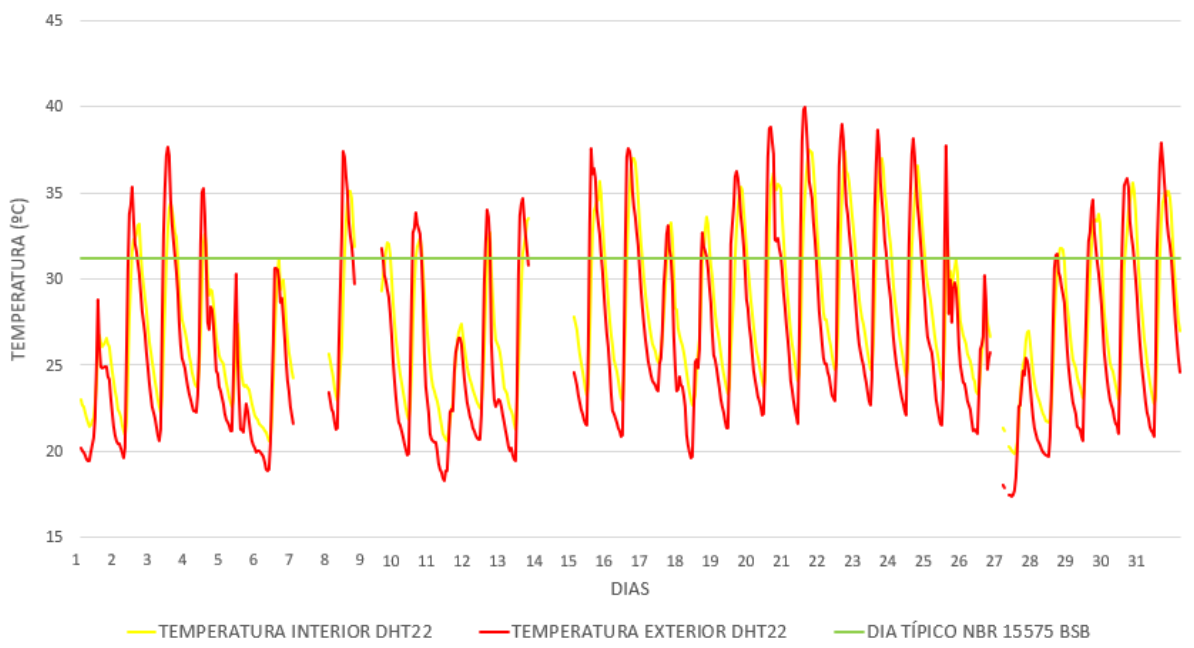

Figura 6 - Temperatura Interna e Externa e Limite Normativo -

Fonte: Autores

UMIDADE RELATIVA - MEDIÇÕES DHT22 E LIMITE ASHRAE 160

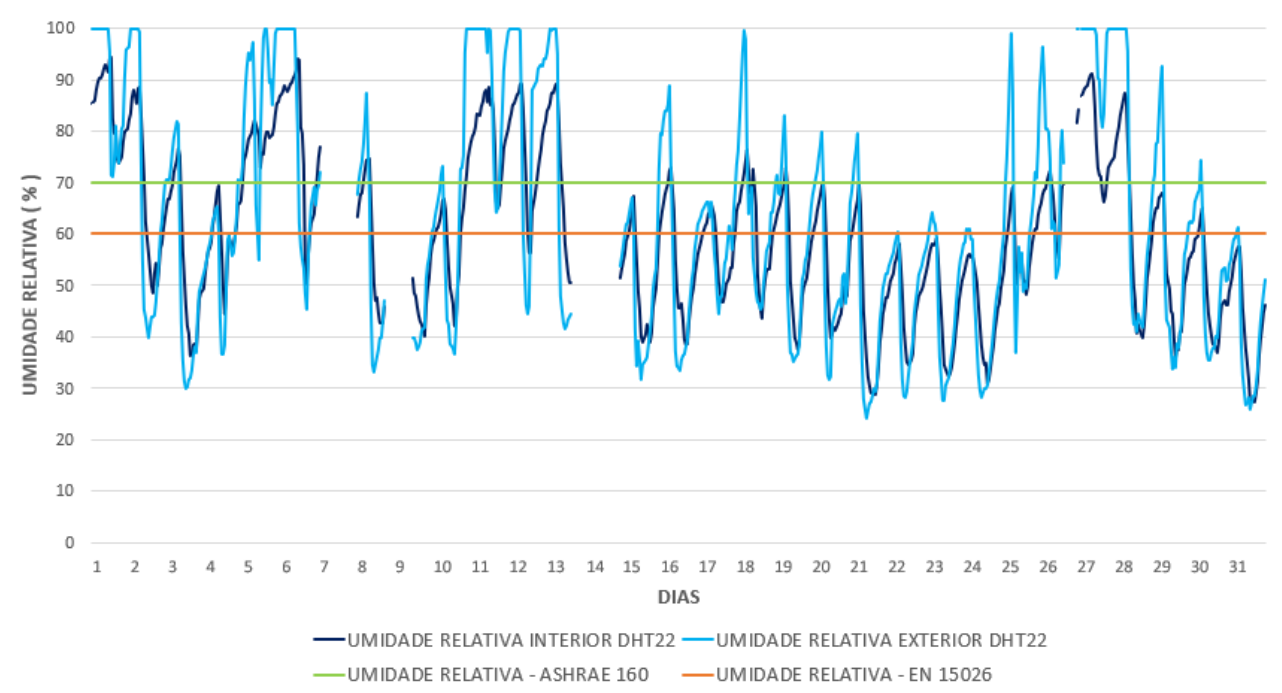

Figura 7 - Medições de umidade com DHT22 -

Fonte: Autores 
Os valores de temperatura medidos externamente variaram entre $17,38^{\circ} \mathrm{C}$ a $39,97^{\circ} \mathrm{C}$, enquanto a umidade relativa obteve valores no intervalo entre $24,2 \%$ e $99,9 \%$, estando próximos dos valores obtidos na simulação. Internamente, a temperatura variou entre $19,82^{\circ} \mathrm{C}$ e $37,47^{\circ} \mathrm{C}$ e a umidade relativa variou entre $27,4 \%$ e $94,53 \%$.

A NBR 15575 estabelece que para a Zona Bioclimática 4 a temperatura interna deve ser sempre menor ou igual à temperatura externa. No entanto, em algumas horas do mês, a temperatura interna foi maior do que a externa. Ainda baseado na NBR 15575, considerando que a temperatura do dia típico de verão para Brasília é de $31,2^{\circ} \mathrm{C}$, observa-se que a temperatura ultrapassa esse limite em diversos momentos horários ao longo do mês.

A NBR 15575 não estabelece valores de referência para a umidade relativa interna. As normas ASHRAE 160 e EM 15026, estabelecem os limites de $70 \%$ e $60 \%$, respectivamente. Observa-se na Figura 7 que, em ambos os casos, a umidade relativa superficial medida ultrapassa os limites estipulados.

Nota-se que os picos de umidade relativa interior coincidem com os de umidade relativa exterior. Isso indica que o sistema construtivo da envoltória, à medida que recebe a chuva dirigida, permite um alto fluxo de umidade para o interior da edificação.

\section{CONCLUSÕES}

Nessa pesquisa foi realizada simulações e medições em campo para avaliar o comportamento higrotérmico para o mês de janeiro da fachada Leste de uma autoconstrução de tijolos cerâmicos revestidos com argamassa, localizada em Santa Maria- DF.

As medições em campo realizadas no ambiente externo obtiveram valores próximos aos simulados. Diferentemente, os valores internos de umidade e temperatura medidos em campo variaram significativamente em relação aos simulados. As diferenças entre os valores simulados e medidos internamente sugerem que a parede da autoconstrução permite um alto fluxo de umidade para $o$ interior da edificação e que pode ser responsável pelo desenvolvimento do mofo encontrado internamente. Os valores de temperatura interna medidos em campo indicam variações além dos limites recomendados pelas normas brasileiras e internacionais.

Visto isso, recomenda-se que as companhias habitacionais encarregadas de realizarem assistência técnica considerem avaliações e monitoramento do desempenho higrotérmico de habitações autoconstruídas e de habitações de interesse social.

\section{REFERÊNCIAS}

AMERICAN SOCIETY OF HEATING, REFRIGERATING AND AIR-CONDITIONING ENGINEERS. Criteria for Moisture-Control Design Analysis in Buildings. ASHRAE 160. Atlanta. 2016.

ASSOCIAÇÃO BRASILEIRA DE NORMAS TÉCNICAS. Norma de Desempenho de Edificações Habitacionais. NBR 15575. Brasília. 2013.

CONSELHO DE ARQUITETURA E URBANISMO /BR. Pesquisa CAU/BR Datafolha. 2015. Disponível em: <https://www.caubr.gov.br/pesquisa2015/>. 
COELHO, G.; SILVA, H. E.; HENRIQUES, F. Calibrated hygrothermal simulation models for historical buildings. Building and Environment. v.142. p. 439-450. Set. 2018.

EUROPEAN STANDARDS. German Institute for Standardization. Hygrothermal performance of building components and building elements - Assessment of moisture transfer by numerical simulation. DIN EN 15026. Berlin. p. 26. 2007.

ZANONI, $V$. Influência dos agentes climáticos de degradação no comportamento higrotérmico de fachadas em Brasília. 2015. 313 f. Tese de doutorado em Arquitetura e Urbanismo. PPG FAU-UnB. Universidade de Brasília, Brasília. 